\title{
MicroRNA-223 promotes osteoblast differentiation of MC3T3-E1 cells by targeting histone deacetylase 2
}

\author{
JIANMING CHEN $^{1,2}$, GUISONG HE ${ }^{3}$, YI WANG ${ }^{4}$ and DAOZHANG CAI ${ }^{1}$ \\ ${ }^{1}$ Department of Orthopedics, The Third Affiliated Hospital of Southern Medical University, Guangzhou, \\ Guangdong 510630; ${ }^{2}$ Department of Orthopedics, The Central Hospital of Yongzhou, Yongzhou, Hunan 425000; \\ ${ }^{3}$ Department of Orthopedic Surgery, The Second Affiliated Hospital of Guangzhou Medical University, \\ Guangzhou, Guangdong 510260; ${ }^{4}$ Department of Otolaryngology/Head and Neck Surgery, \\ The Central Hospital of Yongzhou, Yongzhou, Hunan 425000, P.R. China
}

Received February 21, 2018; Accepted November 19, 2018

DOI: $10.3892 /$ ijmm.2018.4042

\begin{abstract}
MicroRNAs (miRNAs) have emerged as pivotal regulators in various physiological and pathological processes at the post-transcriptional level, and may serve important roles in osteogenic differentiation. However, their roles and functions are not fully understood. In the present study, miR-223-5p was identified as a modulator of osteoblastic differentiation in MC3T3-E1 cells. Reverse transcription-quantitative polymerase chain reaction and western blotting demonstrated that miR-223-5p was significantly upregulated in MC3T3-E1 cells following the induction of osteoblast differentiation. Overexpression of miR-223-5p promoted osteogenic differentiation both in vitro and vivo. Expression of histone deacetylase 2 (HDAC2), which acts as a negative regulator of osteogenesis, was regulated by miR-223-5p. Collectively, the results of the present study revealed a novel miR-223-5p/HDAC2 axis that regulates osteoblast differentiation, and may serve as a potential target for enhancing bone formation in vivo.
\end{abstract}

\section{Introduction}

Osteoblasts are responsible osteogenesis and bone regeneration. The balance between osteoblasts and osteoclasts is crucial for the maintenance of normal morphology and bone tissue strength, in which osteogenic differentiation from mesenchymal stem cells serves an important role $(1,2)$. A previous study demonstrated that disturbances in osteogenic differentiation leads to bone loss (3). This may result in health problems such as osteoporosis (OP) and osteoarthritis (4). Furthermore, bone defects following trauma due to the inhibition of osteogenic

Correspondence to: Professor Daozhang Cai, Department of Orthopedics, The Third Affiliated Hospital of Southern Medical University, 183 West Zhongshan Avenue, Guangzhou, Guangdong 510630, P.R. China

E-mail: daozhang@medmail.com.cn

Key words: microRNA-223-5p, osteogenic differentiation, histone deacetylase 2, MC3T3-E1 cells differentiation severely compromise patient quality of life (5). Therefore, understanding the molecular mechanisms associated with osteogenic differentiation is essential for the treatment of osteogenic disorders.

Non-protein coding RNAs encoded by the human genome are known to be important regulatory transcripts associated with multiple biological processes and pathologies (6). Among them, microRNAs (miRNAs/miRs) are small non-coding RNAs composed of 1-25 nucleotides. miRNAs regulate the expression of multiple genes by disrupting mRNA stability and/or inhibiting translation in conjunction with the RNA-induced silencing complex (6). An increasing number of miRNAs have emerged as pivotal regulators of osteoblast differentiation. miR-34a has been identified as an inhibitor of human stromal cell differentiation through targeting Jagged 1 and downregulating Notch signaling, resulting in bone formation abnormalities (7). miR-628-3p has been shown to regulate osteoblast differentiation by targeting runt-related transcription factor 2 (RUNX2), the master osteoblast transcription factor (8). miR-375 serves as a regulator of osteogenic differentiation in human adipose-derived stem cells, and overexpression of miR-375 promotes osteogenic differentiation via the Yes-associated protein 1/DEP domain containing mTOR-interacting protein/protein kinase B regulatory network (9). These findings highlight the essential role of miRNAs in the process of osteoblast differentiation. However, the number of miRNAs regulating osteoblast differentiation is predicted to be large, and further research focusing on the role of miRNAs in the regulatory mechanisms of osteogenesis is required.

miR-223 participates in multiple biological processes, including myocardial infarction, inflammation and cancer development (10-14). miR-223 may protect myocardial cells from hypoxia-induced apoptosis and excessive autophagy by targeting poly(ADP-ribose) polymerase 1 (10). In addition, the miR-223-5p/-3p duplex has been verified to cooperatively inhibit ischemia/reperfusion-induced cardiac necroptosis (11). miR-223 is involved in the regulation of neutrophil wound response and nuclear factor- $\kappa \mathrm{B}$ activation by directly targeting Cullin $1 \mathrm{a} / \mathrm{b}$, tumor necrosis factor receptor-associated factor 6 , and transforming growth factor- $\beta$-activated kinase 1 
(MAP3K7)-binding protein 1 (12). miR-223-5p inhibits the migration and invasion of bladder cancer cells by regulating anillin actin-binding protein (13). Decreased miR-223 levels abate the osteogenic differentiation potential of bone marrow mesenchymal stromal cells derived from patients with multiple myeloma (14), suggesting that that miR-223 may serve as a regulator of osteogenic differentiation.

The present study demonstrated that miR-223-5p has critical function in osteoblast differentiation. miR-223-5p was upregulated during consecutive osteogenic induction, and promoted osteogenic differentiation. Furthermore, histone deacetylase 2 (HDAC2) was identified as a target of miR-223-5p, and downregulation of HDAC2 by miR-223-5p induced osteoblast differentiation. Therefore, miR-223-5p may represent a potential therapeutic target for bone regeneration-associated diseases.

\section{Materials and methods}

Cell culture and osteogenic differentiation. MC3T3-E1 cells were obtained from the American Type Culture Collection (Manassas, VA, USA) and routinely maintained in growth medium (GM) consisting of $\alpha$-modified Eagle's medium $(\alpha$-MEM) supplemented with $10 \%$ fetal bovine serum (both Gibco; Thermo Fisher Scientific, Inc., Waltham, MA, USA), penicillin $(50 \mathrm{U} / \mathrm{ml})$ and streptomycin $(50 \mu \mathrm{g} / \mathrm{ml})$ at $37^{\circ} \mathrm{C}$ in a humidified atmosphere with $5 \% \mathrm{CO}_{2}$. To induce osteogenic differentiation, the cells were incubated with osteogenic medium (OM), containing $10 \mathrm{ng} / \mathrm{ml} \beta$-glycerophosphate (Sigma-Aldrich; Merck KGaA, Darmstadt, Germany), $10^{-7} \mathrm{mmol} / 1$ dexamethasone (Sigma-Aldrich; Merck KGaA), and $50 \mu \mathrm{g} / \mathrm{ml}$ vitamin C (Sigma-Aldrich; Merck KGaA) for 7 or 14 days. Next, the induced cells were digested for further detection. For the CAY10683 treatment, CAY10683 (Selleck Chemicals, Houston, TX USA) was added to the OM at concentrations of $0.01,0.1$ and $1 \mu \mathrm{M}$ for 7 days. CAY10683 was dissolved in dimethyl sulfoxide (Sigma-Aldrich; Merck KGaA).

Cell transfection. miR-223-5p mimics, inhibitor and their corresponding negative controls (NCs) were purchased from Guangzhou RiboBio Co., Ltd. (Guangzhou, China). The sequences were as follows: miR-223-5p mimic, 5'-CGUGUA UUUGACAAGCUGAGUUG-3'; miR-223-5pinhibitor,5'-CAA CUCAGCUUGUCAAAUACACG-3'; mimics-NC, 5'-UUU GUACUACACAAAAGUACUG-3'; and inhibitor-NC, 5'-UUU GUACUACACAAAAGUACUG-3'. Small interfering (si)RNAs against HDAC2 (5'-AAGCCUCAUAGA AUCCGCAUG-3') and plasmid overexpressing HDAC2 (HDAC2-pcDNA3.1) were purchased from Shanghai GenePharma Co., Ltd. (Shanghai, China). MC3T3-E1 cells were cultured in six-well plates at a concentration of $2 \times 10^{5}$ cells/well. When cells reached $70-80 \%$ confluence, mimics $(50 \mathrm{nM})$, inhibitor $(25 \mathrm{nM})$, siRNAs $(25 \mathrm{nM})$ or plasmids $(2.5 \mu \mathrm{g} / \mathrm{ml})$ were transfected into cells using Lipofectamine ${ }^{\circledR} 3000$ (Invitrogen; Thermo Fisher Scientific, Inc.) according to the manufacturer's protocol. After $48 \mathrm{~h}$ incubation, cells were subjected to further experiments.

$R N A$ isolation and reverse transcription-quantitative polymerase chain reaction ( $R T-q P C R)$. Total RNA was extracted with TRIzol ${ }^{\circledR}$ reagent (Invitrogen; Thermo Fisher
Scientific, Inc.) and the reverse transcription reaction was performed with $1 \mu \mathrm{g}$ total RNA using a PrimeScript ${ }^{\mathrm{TM}}$ RT reagent kit according to the manufacturer's protocol. RT-qPCR was conducted with SYBR-Green I (both Takara Bio, Inc., Otsu, Japan) on the LightCycler 480 II system (Roche Diagnostics, Basel, Switzerland). The thermocycling conditions were as follows: $95^{\circ} \mathrm{C}$ for $30 \mathrm{sec}$, followed by 36 cycles of denaturation at $95^{\circ} \mathrm{C}$ for $15 \mathrm{sec}$, annealing at $60^{\circ} \mathrm{C}$ for $15 \mathrm{sec}$ and extension at $72^{\circ} \mathrm{C}$ for $15 \mathrm{sec}$. Primer sequences were as follows: miR-223-5p, 5'-CGCGCGTGTATTTGACA AGC-3', and 5'-AGTGCAGGGTCCGAGGTATT-3'; U6, 5'-CTCGCTTCGGCAGCACA-3', and 5'-AACGCTTCACGA ATTTGCGT-3'; Alkaline phosphatase (ALP), 5'-TGGCTC TGCCTTTATTCCCTAGT-3', and 5'-AAATAAGGTGCT TTGGGAATCTGT-3'; Osteocalcin (OCN), 5'-GCCATCA CCCTGTCTCCTAA-3', and 5'-GCTGTGGAGAAGACACA CGA-3'; RUNX2, 5'-GCCGGGAATGATGAGAACTA-3', and 5'-GGTGAAACTCTTGCCTCGTC-3'; HDAC2, and 5'-GCTATTCCAGAAGATGCTGTTC-3', 5'-GTTGCTGAG CTGTTCTGATTTG-3'; $\beta$-actin, 5'-TCACCCACACTGTGCC

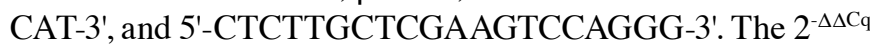
method was used to quantify mRNA and miRNA expression. Data were normalized to $\beta$-actin (mRNA) or U6 (miRNA).

Measurement of alkaline phosphatase (ALP) activity. Cultured cells were rinsed in PBS three times, and total protein was extracted using radioimmunoprecipitation assay (RIPA) lysis buffer (Sigma-Aldrich; Merck KGaA) and quantified with a bicinchoninic acid (BCA) protein assay. ALP activity was measured with the Alkaline Phosphatase, Diethanolamine Detection kit (cat. no. AP0100; Sigma-Aldrich; Merck KGaA). Equal volumes of cell lysate (50 $\mu \mathrm{l})$ were added to each well of the 96-well plates, and incubated with an ALP staining solution at $37^{\circ} \mathrm{C}$ for $1 \mathrm{~h}$. Following the addition of stop solution, ALP activity was measured spectrophotometrically at $405 \mathrm{~nm}$, and normalized to total protein concentration.

Alizarin Red S staining. Mineralization was determined by Alizarin red S staining. Cells were plated in six-well plates ( $5 \times 10^{5}$ cells/well), fixed with $4 \%$ paraformaldehyde for $10 \mathrm{~min}$ at room temperature and stained with $0.1 \%$ Alizarin red staining solution ( $\mathrm{pH}$ 4.2; Sigma-Aldrich; Merck KGaA) at room temperature for $20 \mathrm{~min}$. The cells were washed with PBS, and images were captured under a light microscope (x200).

Western blotting. The cells were harvested and lysed with RIPA lysis buffer containing phenylmethane sulfonyl fluoride and protease inhibitors (both Sigma-Aldrich; Merck KGaA). The concentration of each sample was determined by a BCA protein assay. Equal amounts of protein (30 $\mu \mathrm{g} / \mathrm{lane})$ were loaded and separated by $10 \%$ SDS-PAGE, followed by transfer onto $0.4 \mu \mathrm{m}$ polyvinylidene difluoride membranes (EMD Millipore, Billerica, MA, USA). The membranes were blocked with 5\% non-fat milk at room temperature for $1 \mathrm{~h}$, and incubated with different primary antibodies overnight at $4^{\circ} \mathrm{C}$. The primary antibodies included anti-ALP rabbit polyclonal antibody (1:1,000; cat. no. 11187-1-AP; ProteinTech Group, Inc., Chicago, IL, USA), anti-OCN rabbit polyclonal antibody (1:500; cat. no. 23418-1-AP; ProteinTech Group, Inc.), anti-RUNX2 rabbit polyclonal antibody (1:800; 
A
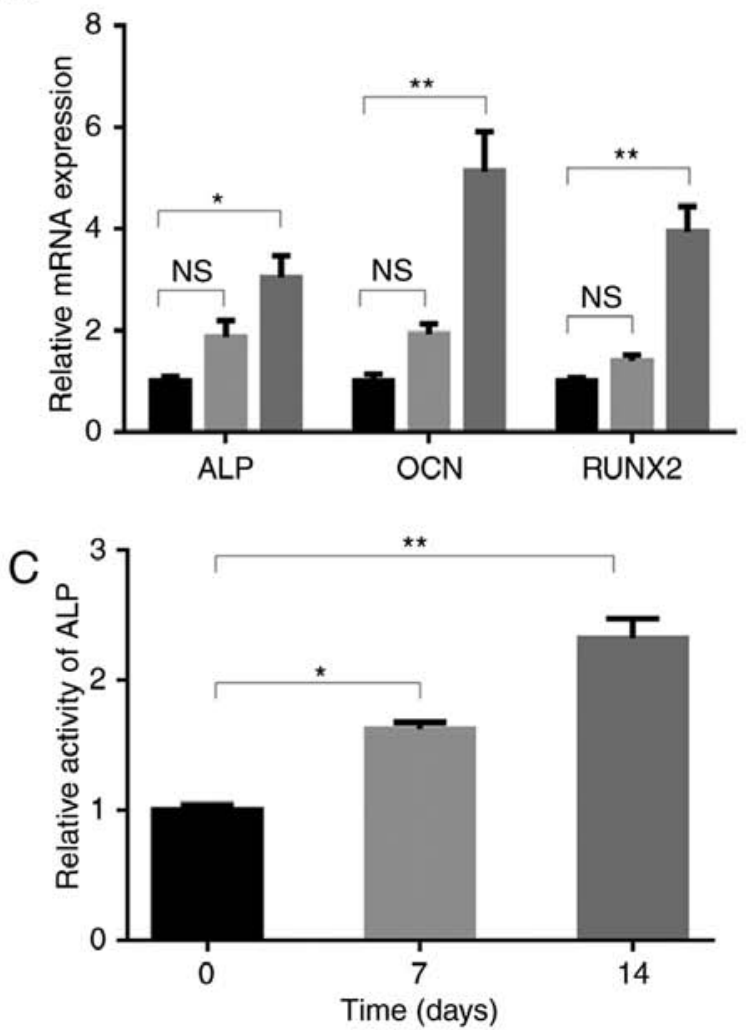

B
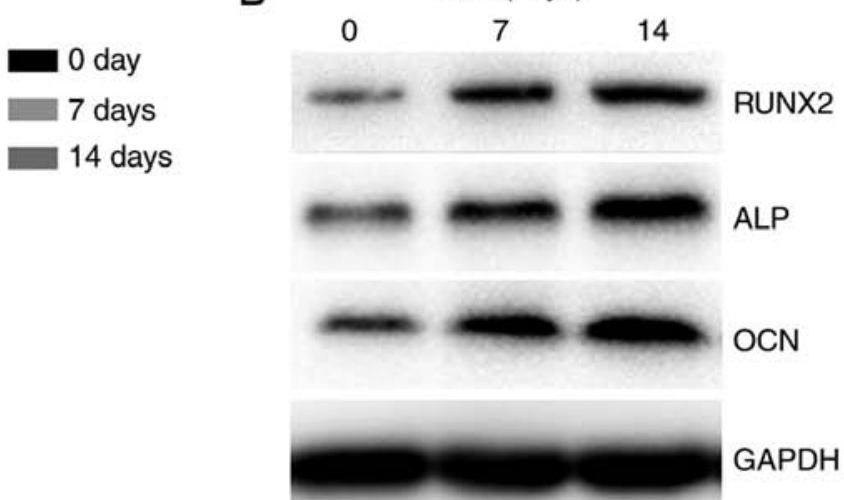

ALP
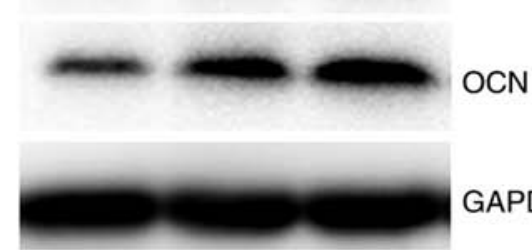

GAPDH

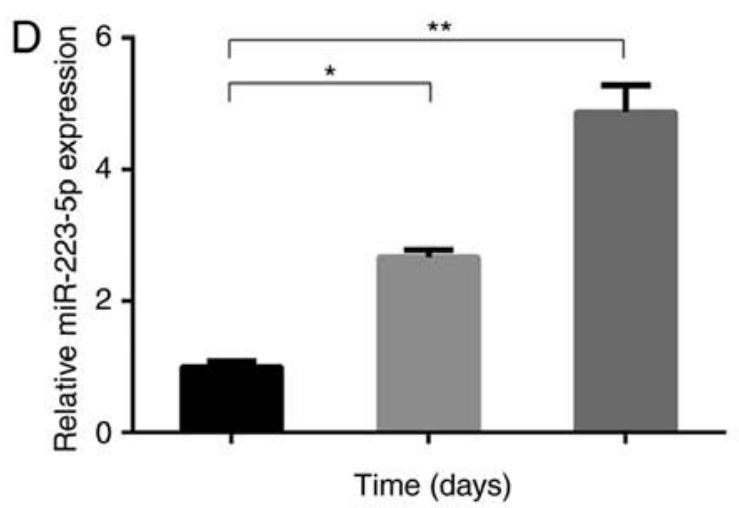

Figure 1. miR-223-5p is upregulated in osteogenic differentiation. (A) Osteogenesis-associated gene and (B) protein expression, including ALP, OCN and RUNX2, in MC3T3-E1 cells following treatment with OM for 0, 7 and 14 days. (C) ALP activity and (D) the expression of miR-223-5p was assayed by RT-qPCR at 0,7 and 14 days after OM treatment. ${ }^{*} \mathrm{P}<0.05,{ }^{* *} \mathrm{P}<0.01$. OM, osteogenic medium; miR, microRNA; RT-qPCR, reverse transcription-quantitative polymerase chain reaction; ALP, alkaline phosphatase; OCN, osteocalcin; RUNX2, runt-related transcription factor 2; NS, not significant.

cat. no. 12556; Cell Signaling Technology, Inc., Danvers, MA, USA), anti-HDAC2 rabbit polyclonal antibody $(1: 1,000$; cat. no. 12922-3-AP; ProteinTech Group, Inc.), and anti- $\beta$-actin rabbit polyclonal antibody (1:2,000; cat. no. 20536-1-AP; ProteinTech Group, Inc.). Membranes were next probed with horseradish peroxidase-conjugated anti-rabbit secondary antibody (1:5,000; cat. no. 7074; Cell Signaling Technology, Inc.), and protein signals were obtained with enhanced chemiluminescence plus substrate (EMD Millipore).

In vivo transplantation. All animal procedures were approved by the Animal Care Committee of Southern Medical University. Healthy female NOD/SCID mice (5 weeks old; $\sim 20 \mathrm{~g}$ ) were purchased from Guangdong Medical Laboratory Animal Center (Guangdong, China) were randomly divided into two groups, with six mice per group. Transfected cells $\left(5 \times 10^{6}\right)$ were loaded onto $20 \mathrm{mg}$ hydroxyapatite-tricalcium phosphate scaffold (HA-TCP; Sigma-Aldrich; Merck KGaA) and subcutaneously implanted into the dorsal region of NOD/SCID mice under anesthesia. After 4 weeks, xenografts were removed, fixed with $4 \%$ paraformaldehyde for 2 days at room temperature and decalcified in 10\% EDTA ( $\mathrm{pH} 6.0$ ) for another 7 days at room temperature. Xenografts were then embedded in paraffin, sectioned at $4 \mu \mathrm{m}$ thickness and stained with hematoxylin and eosin (H\&E; Beyotime Institute of Biotechnology, Shanghai, China), or Masson's Trichrome stain (Sigma-Aldrich; Merck KGaA), according to the manufacturer's protocols.
Statistical analysis. All data were presented as the mean \pm standard deviation. Statistical analyses were performed with SPSS software, version 19.0 (IBM Corp., Armonk, NY, USA). The significance of mean values between two groups was analyzed using a two-tailed unpaired Student's t-test. Differences in multiple groups were determined by one-way analysis of variance with subsequent Bonferroni correction. $\mathrm{P}<0.05$ was considered to indicate a statistically significant difference.

\section{Results}

miR-223-5p is upregulated in osteogenic differentiation. To investigate the role of $\mathrm{miR}-223-5 \mathrm{p}$ in osteogenic differentiation, the present study detected its dynamic expression profiles in MC3E3T1 cells incubated with osteogenic inducers. The expression levels of osteogenesis-associated genes, including ALP, OCN and RUNX2 were significantly upregulated under differentiation-inducing conditions. On day 14, they had increased by 3 (ALP), 5.1 (OCN) and 3.9 (RUNX2) fold, compared with day 0 (Fig. 1A). The upregulation of RUNX2, ALP and OCN protein was also confirmed by western blotting (Fig. 1B). Additionally, the activity of ALP, an indicator of mineralization induced by osteogenic differentiation, was promoted following induction (Fig. 1C). Furthermore, miR-223-5p expression in MC3T3-E1 cells gradually increased during osteogenic differentiation, reaching a $>4$ fold increase at day 14, compared with day 0 (Fig. 1D), indicating the potential involvement of miR-223-5p in osteogenic induction. 

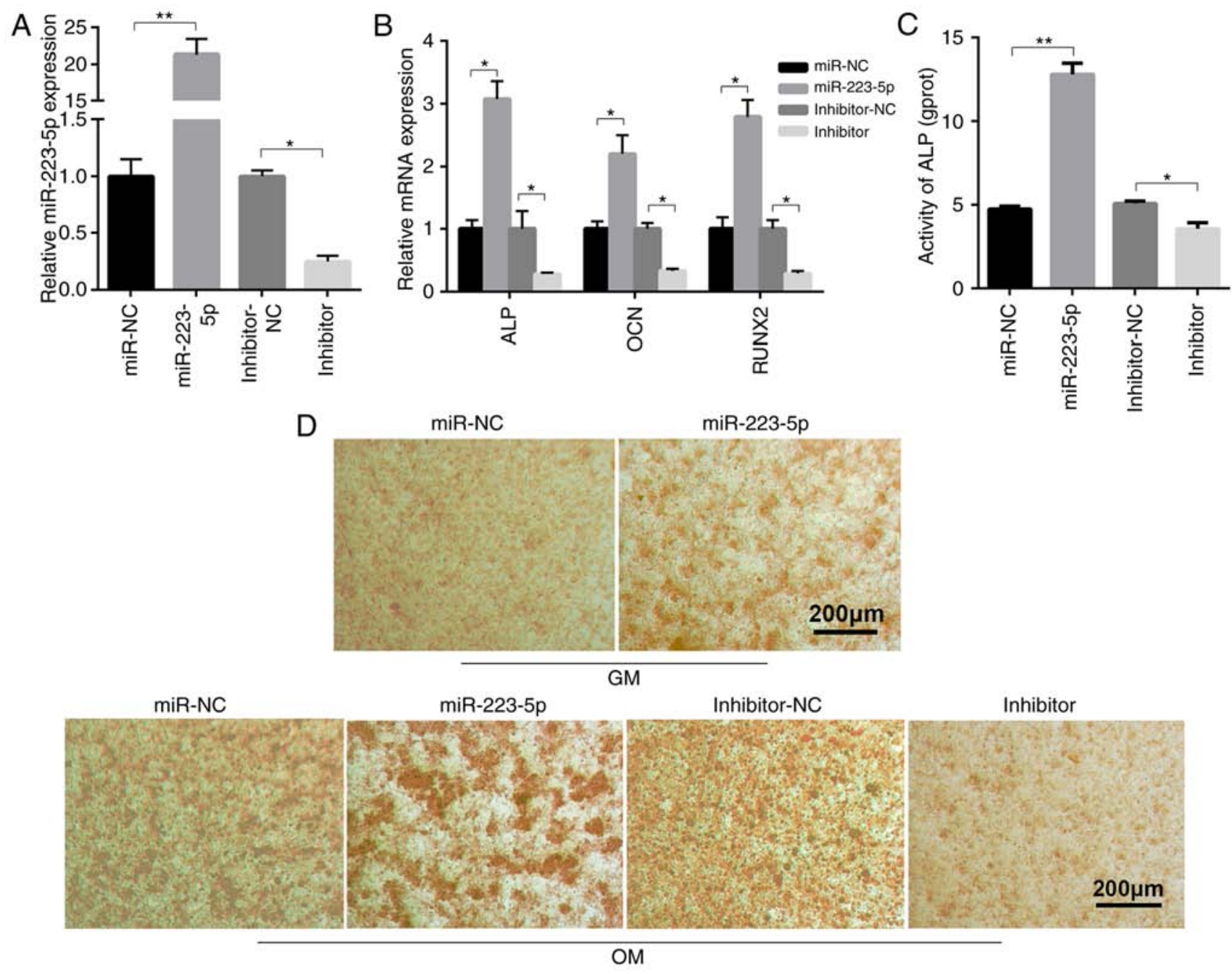

Figure 2. miR-223-5p promotes osteogenic differentiation. (A) miR-223-5p expression was validated in MC3T3-E1 cells transfected with miR-223-5p mimics or inhibitor and their corresponding controls by RT-qPCR. (B) ALP, OCN and RUNX2 expression and (C) ALP activity was measured in transfected MC3T3-E1 cells after 7 days of OM treatment. (D) Alizarin red $\mathrm{S}$ staining was also performed after 7 days of $\mathrm{OM}$ treatment. ${ }^{*} \mathrm{P}<0.05$, $^{* *} \mathrm{P}<0.01$. miR, microRNA; $\mathrm{ALP}$, alkaline phosphatase; OCN, osteocalcin; RUNX2, runt-related transcription factor 2; OM, osteogenic medium; RT-qPCR, reverse transcription-quantitative polymerase chain reaction.

miR-223-5p contributes to osteogenic differentiation and mineralization. To clarify the biological roles of miR-223-5p in osteogenic differentiation, miR-233-5p overexpression and inhibition was induced in MC3T3-E1 cells. The effect of miR-223-5p and HDAC2 on osteogenic differentiation can be detected in 7 days OM treatment. The expression of ALP, OCN and RUNX2 increased in 7 days OM treatment although they had no statistical significance. While miR-223-5p treatment promoted the expression of ALP, OCN and RUNX2 after 7 days of OM treatment, which was confirmed by RT-qPCR. miR-223-5p overexpression and silencing was shown to be successful by RT-qPCR (Fig. 2A). miR-223-5p mimic transfection markedly increased the expression of ossification-associated genes, compared to transfection with negative control (NC) miRNA mimics. In contrast, inhibiting miR-223-5p expression significantly reduced ossification-associated gene expression (Fig. 2B). Further results revealed that ALP activity in the differentiated MC3T3-E1 cells was inhibited by transfection with miR-223-5p mimics, but enhanced by the miR-223-5p inhibitor, compared with the corresponding NC group
(Fig. 2C). The degree of cell mineralization, as determined by Alizarin red S staining, increased following miR-223-5p mimic transfection compared with the control group in MC3T3-E1 cells cultured with either GM or OM medium. miR-223-5p inhibitor reduced osteogenic differentiation, as shown by the notable reduction in mineralization nodules (Fig. 2D). Together, these results indicated that miR-223-5p served an important role in osteogenic differentiation and mineralization.

HDAC2 is a negative target of miR-223-5p and is involved in osteogenic differentiation. It has previously been reported that HDAC2 is a downstream target of miR-223-5p in chronic obstructive pulmonary disease (15), but their relationship in osteogenic differentiation remains to be elucidated. In the present study, it was investigated whether miR-223-5p regulated HDAC2 expression. RT-qPCR and western blotting demonstrated that HDAC2 gene expression was reduced by $70 \%$ of the NC group in the presence of miR-223-5p mimics, and increased $>3$ fold when transfected with miR-223-5p inhibitor, compared with the inhibitor NC group (Fig. 3A). HDAC2 
A

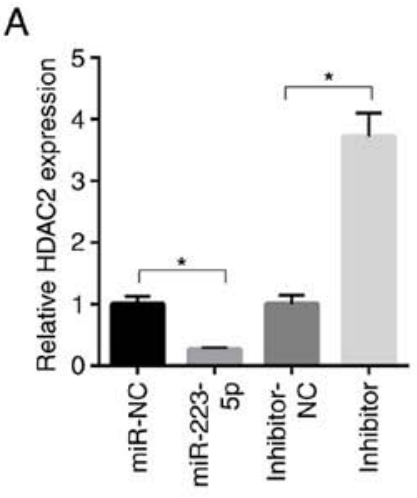

D

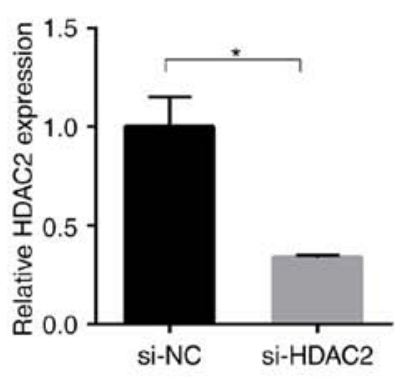

B

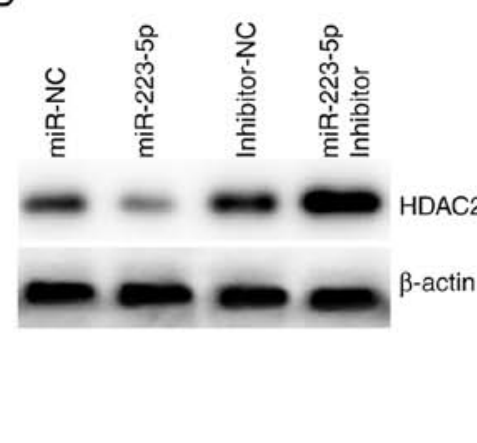

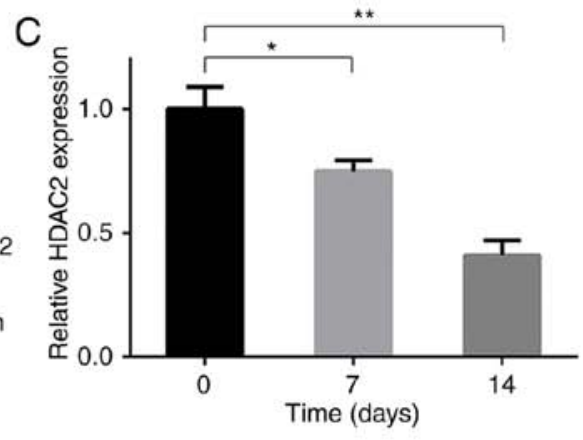

E

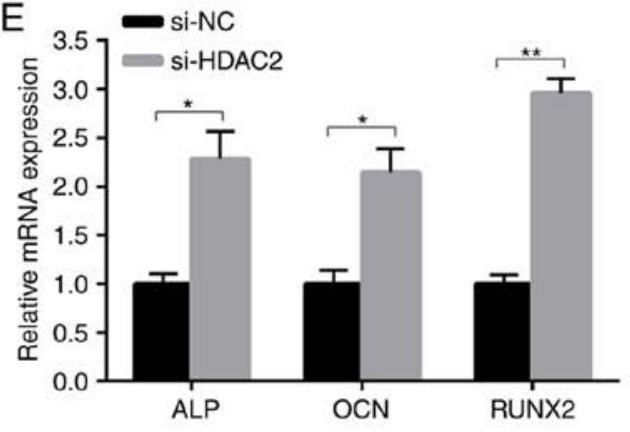

F si- si-

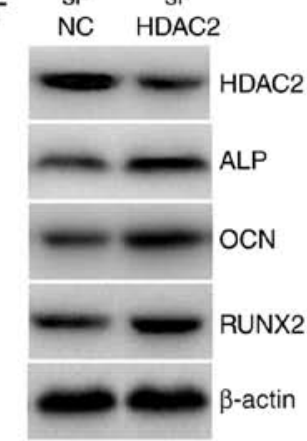

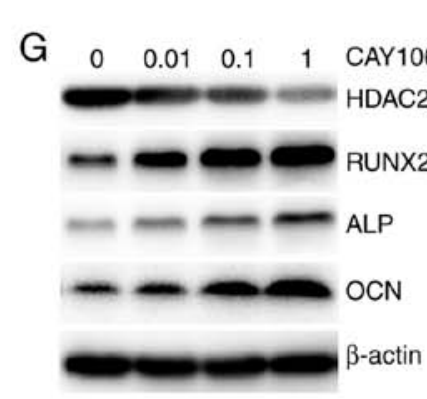

$J$

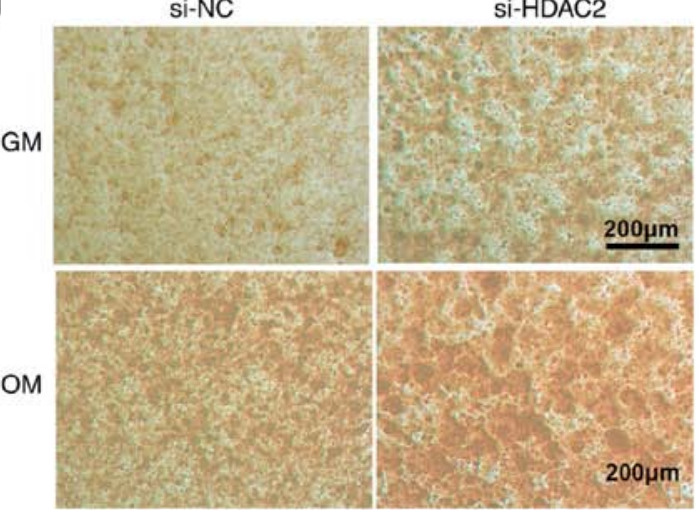

MC3E3T1
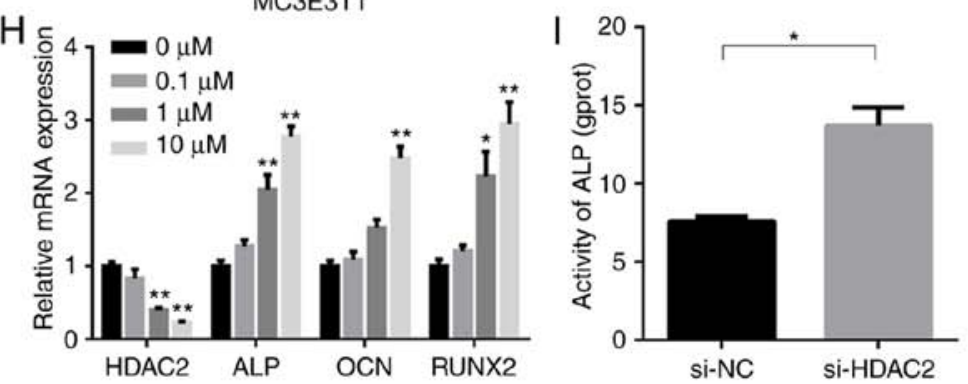

$\mathrm{K}$

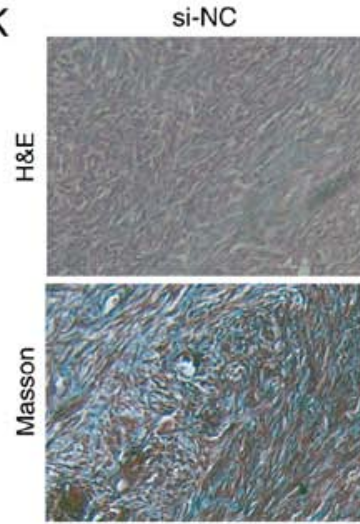

si-HDAC2

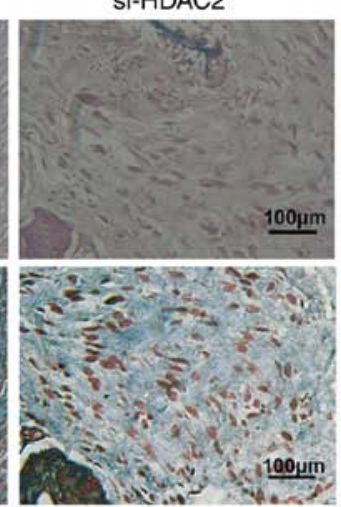

Figure 3. HDAC2 is a target of miR-223-5p and is involved in osteogenic differentiation. (A) The gene and (B) protein expression of HDAC2 was detected in transfected MC3T3-E1 cells. (C) After 0, 7 and 14 days of OM treatment, HDAC2 expression was determined by RT-qPCR. (D) Silencing of HDAC2 expression was validated in MC3T3-E1 cells transfected with si-HDAC2 or NC by RT-qPCR. (E) ALP, OCN and RUNX2 mRNA, as well as (F) ALP, OCN, RUNX2 and HDAC2 protein expression, was detected in MC3T3-E1 cells transfected with si-HDAC2, 7 days after osteogenic induction. *P<0.05, ** $\mathrm{P}<0.01$. (G) ALP, OCN, HDAC2 and RUNX2 mRNA and (H) protein expression was also detected in MC3T3-E1 cells treated with OM and CAY10683 at the indicated concentrations for 7 days. ${ }^{* *} \mathrm{P}<0.01$ vs. $0 \mu \mathrm{M}$ group. (I) ALP activity in si-HDAC2-transfected MC3T3-E1 cells after 7 days of OM treatment. (J) The osteogenic differentiation of MC3T3-E1 cells transfected with si-HDAC2 was determined by Alizarin Red S staining. (K) Osteogenic differentiation of xenografts was determined by H\&E and Masson's Trichrome staining. miR, microRNA; RT-qPCR, reverse transcription-quantitative polymerase chain reaction; HDAC2, histone deacetylase 2; ALP, alkaline phosphatase; OCN, osteocalcin; RUNX2, runt-related transcription factor 2; OM, osteogenic medium; H\&E, hematoxylin and eosin.

protein expression was also decreased in miR-223-5p-overexpressing MC3T3-E1 cells, and increased in MC3T3-E1 cells with downregulated miR-223-5p (Fig. 3B). RT-qPCR revealed that HDAC2 mRNA expression markedly decreased during 


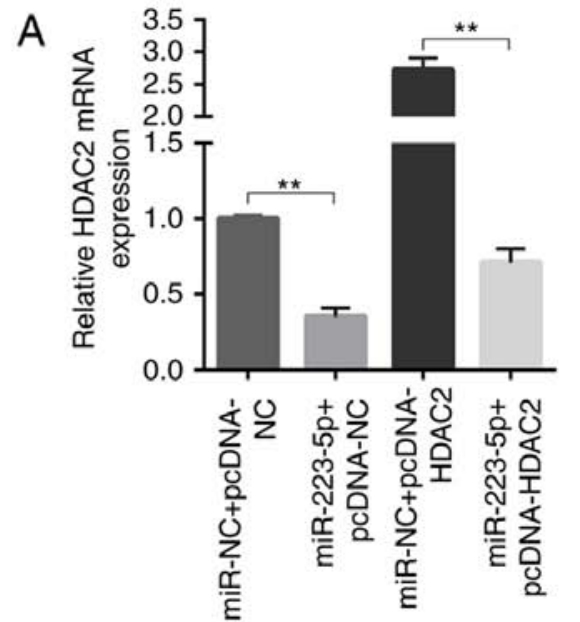

C

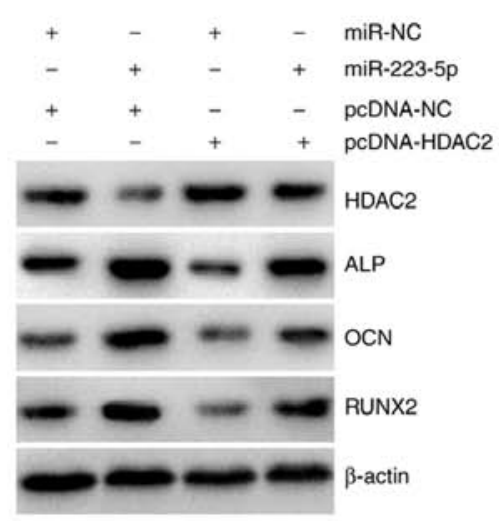

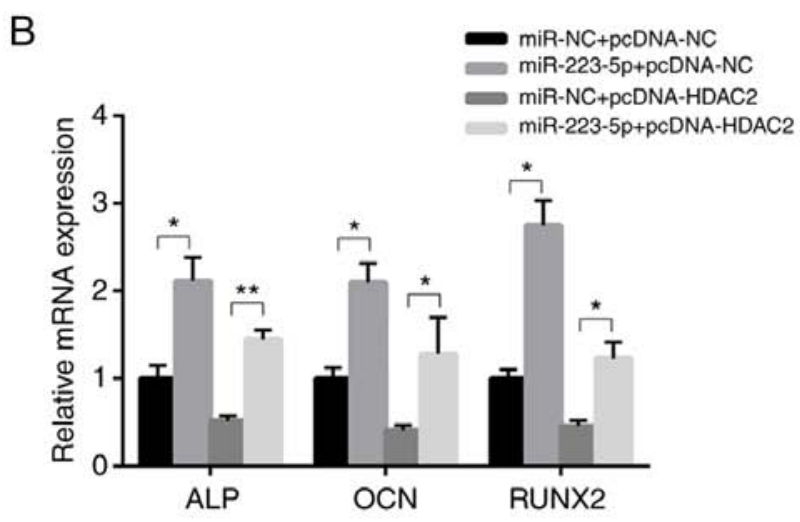

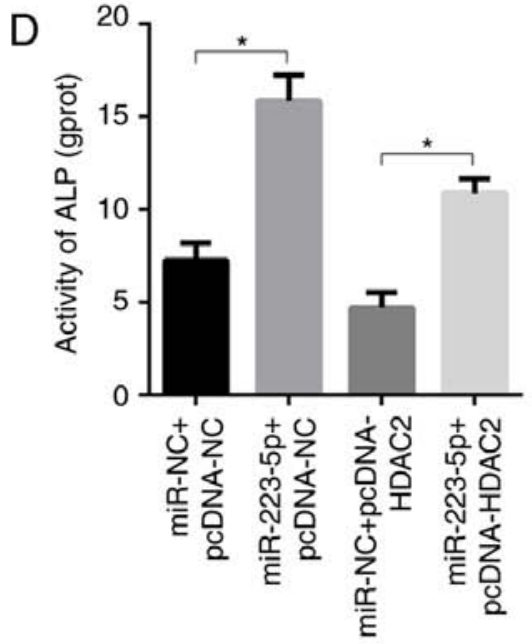

\section{E miR-NC+ \\ miR-223-5p+ pcDNA-NC}

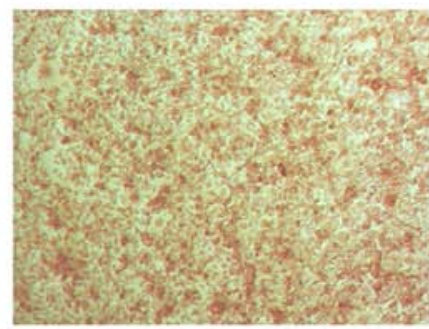
miR-NC+ pcDNA-HDAC2

miR-223-5p+ pcDNA-HDAC2
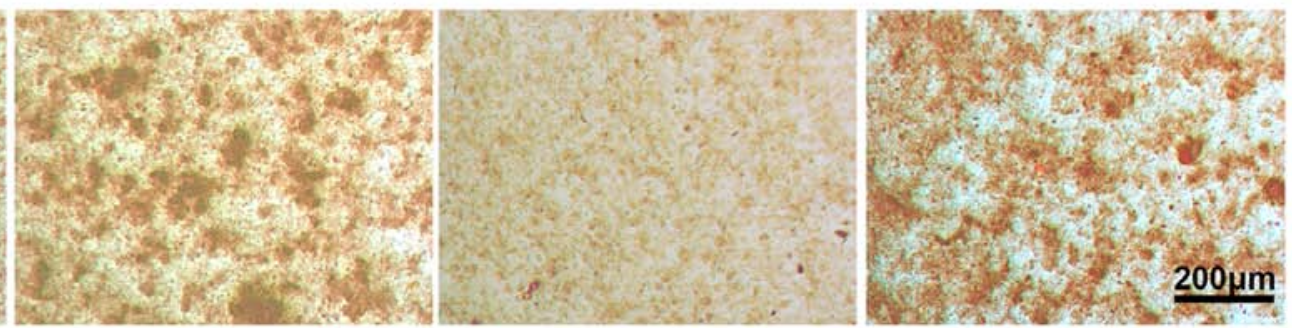

Figure 4. HDAC2 overexpression attenuates the effects of miR-223-5p in osteogenic differentiation. (A) mRNA expression of HDAC2 in MC3T3-E1 cells co-transfected with miR-223-5p mimics and/or pcDNA-HDAC2. (B) The mRNA and (C) protein expression of ALP, OCN and RUNX2 was assessed in MC3T3-E1 cells co-transfected with miR-223-5p mimics and/or pcDNA-HDAC2. (D) ALP activity assay and (E) Alizarin Red S staining of MC3T3-E1 cells co-transfected with miR-223-5p mimics and/or pcDNA-HDAC2. ${ }^{*} \mathrm{P}<0.05,{ }^{* *} \mathrm{P}<0.01$. miR, microRNA; HDAC2, histone deacetylase 2; ALP, alkaline phosphatase; OCN, osteocalcin; RUNX2, runt-related transcription factor 2.

osteoblast differentiation (Fig. 3C). To evaluate the role of HDAC2 in osteoblast differentiation, the present study transfected MC3T3-E1 cells with si-HDAC2 (Fig. 3D). ALP, OCN and RUNX2 mRNA (Fig. 3E) and protein (Fig. 3F) expression was increased in cells transfected with si-HDAC2. Consistently, CAY10683, a HDAC2 inhibitor, induced a dose-dependent decrease in HDAC2 expression, and a consequent increase in osteogenic-associated gene and protein expression (Fig. 3G and $\mathrm{H})$. Furthermore, si-HDAC2 transfection increased ALP activity, compared with the NC group (Fig. 3I). Alizarin red $\mathrm{S}$ staining also revealed that HDAC2 silencing increased mineralized bone matrix formation (Fig. 3J). Next, MC3E3T1 from control groups and si-HDAC2 groups were loaded onto HA-TCP and then implanted into NOD/SCID mice. The results showed that the MC3E3T1 in the si-HDAC2 groups group formed more osteoids than those in the negative control groups (Fig. 3K).

To further determine the role of HDAC2 in miR-223-5p-mediated osteogenic differentiation, HDAC2overexpressing and control groups were established by transfecting pcDNA3.1-HDAC2 plasmid and pcDNA3.1-NC, which were then co-transfected with miR-223-5p mimics or 
mimics-NC (Fig. 4A). The upregulating effects of miR-223-5p on the expression of ossification-associated genes were markedly inhibited by HDAC2, as confirmed by RT-qPCR (Fig. 4B), western blot analysis (Fig. 4C), ALP activity (Fig. 4D) and Alizarin red S staining (Fig. 4E). Collectively, these findings suggested that HDAC2 may be a negative regulator of osteogenic differentiation.

\section{Discussion}

$\mathrm{OP}$ is a common disease, with an incidence of $13.2 \%$ in China (16). The serious complications of OP, including osteoporotic fractures, pain and misshapen bones, are associated with a high cost of treatment and prevention, and pose a major socioeconomic burden (17). Abnormal stimulation of osteoclasts generally results in bone loss, which leads to OP, and this disease is primarily characterized by decreased bone mass and disorders of the bone microstructure (18-20). Current therapies for OP, such as bone resorption inhibitors and bisphosphonate, are mainly focused on the balance of bone remodeling, which is critical during maintenance and regeneration of bone tissue. However, effective methods for promoting bone formation are still under development $(21,22)$. Therefore, novel effective methods for treating OP and promoting bone synthesis are urgently needed.

miRNAs belong to a small RNA family and have important regulatory properties (23). miRNAs play key roles in the physiological processes and in the pathogenic mechanism of numerous diseases $(24,25)$. An increasing number of studies have confirmed the involvement of several miRNAs in the regulation of bone biology; their potential role in osteogenesis has been reported, but the relative functional significance is not yet completely understood (5,7,26-28). A recent study using miRNA microarray chip technology demonstrated that miR-223 is abnormally expressed in patients with diffuse idiopathic skeletal hyperostosis (DISH), which is associated with sclerostin metabolism (29). DISH is characterized by new bone formation, constitutional abnormalities and metabolic abnormalities, leading to the biomechanical alterations in the musculoskeletal system and/or the formation of obstructive cervical masses $(30,31)$. However, the function of miR-223 in osteoblasts remains unknown.

To the best of our knowledge, the present study is the first to reveal that miR-223-5p was upregulated during osteoblast differentiation. miR-223-5p expression increased gradually in MC3T3-E1 cells over a 14 day period. Accordingly, the expression levels of ALP, OCN and RUNX2 were all significantly upregulated in a time-dependent manner. ALP (32), OCN (33) and RUNX2 (27) are classic biomarkers, that reflect the functional status of osteoblast cells. The capacity of each factor in regulating osteogenic differentiation is highly associated with the bone formation process due to of the dynamic feedback system in the human body. The expression of miR-223-5p exhibited a similar tendency in the present study, suggesting that miR-223-5p may be used as a biomarker for diagnostic purposes for osteogenic differentiation. However, the mechanisms underlying the role of miR-223-5p in this process has not yet been fully elucidated.

The present study demonstrated that miR-223-5p was a positive regulator of osteogenic differentiation, as its overexpression led to the enhancement of osteogenic differentiation, and its silencing had to the opposite effect. Following transfection of miR-233 mimics, biomarkers of osteogenic differentiation (ALP, OCN and RUNX2) were upregulated at 7 days, and the Alizarin $\mathrm{S}$ red staining results revealed a higher number of mineralized nodules compared with the control group. The transfection of miR-233 inhibitor exerted the opposite effects. In the animal experiment, the newly formed bone tissue stained red with H\&E and stained blue with Masson's Trichrome. The area of new bone tissue was larger in implants containing si-HDAC2 cells, compared with negative controls, indicating that HDAC2 silencing increased mineralized bone matrix formation. These results indicated that miR-223-5p may be a target for the treatment of bone loss and the optimization of fracture healing. Several miRNAs including miR-5100 (34), miR-192 (22) and miR-10a (5), have been proven to act as regulatory factors, as their expression markedly affect osteogenic differentiation. Accumulating evidence highlights the crucial role of miRNAs during osteogenic differentiation, and these may exert their effects through targeting their downstream genes. miR-590, miR-9 and miR-5100 control osteogenic differentiation by targeting mothers against decapentaplegic homolog 7, Dickkopf WNT Signaling Pathway Inhibitor 1 and various other genes $(27,35,36)$. However, the target genes of miR-223-5p in osteogenic differentiation remain unknown.

The present study demonstrated that miR-223-5p promoted osteogenic differentiation, at least in part by targeting HDAC2. The experimental data demonstrated that miR-223-5p downregulated HDAC2 gene expression, which has been described as an anti-proliferative gene involved in cell cycle regulation $(37,38)$. A study of chronic obstructive pulmonary disease (COPD) demonstrated that miR-223-5p overexpression decreased HDAC2 expression in human pulmonary artery endothelial cells, whereas HDAC2 expression was preserved when miR-223-5p was silenced (15). These findings suggest that miR-223-5p controls the expression of HDAC2 in COPD (15). Thus, it is probable that miR-223-5p controlled the expression of HDAC2 in osteoblasts, which is a novel regulatory axis.

In conclusion, HDAC 2 expression at the mRNA and protein level was altered following transfection with miR-223-5p mimics or inhibitor in the present study. Therefore, it was demonstrated that miR-223-5p regulated HDAC2 expression to promote osteogenesis. Taken together, the results of the present study indicated a novel potential therapeutic approach to the treatment of osteogenic conditions.

\section{Acknowledgements}

Not applicable.

\section{Funding}

No funding was received.

\section{Availability of data and materials}

All data generated or analyzed during this study are available from the corresponding author upon reasonable request. 


\section{Authors' contributions}

JC contributed the central idea, analyzed the majority of the data and wrote the initial draft of the manuscript. GH and YW performed the in vivo experiments. DC contributed to study design, revised and finalized the manuscript. All authors read and approved the final manuscript.

\section{Ethics approval and consent to participate}

The research protocols associated with the experimental mice were approved by the Experimental Animal Ethics Committee of Southern Medical University.

\section{Patient consent for publication}

Not applicable.

\section{Competing interests}

The authors declare that they have no competing interests.

\section{References}

1. Kular J, Tickner J, Chim SM and Xu J: An overview of the regulation of bone remodelling at the cellular level. Clin Biochem 45: 863-873, 2012.

2. Wei J, Shi Y, Zheng L, Zhou B, Inose H, Wang J, Guo XE, Grosschedl R and Karsenty G: miR-34s inhibit osteoblast proliferation and differentiation in the mouse by targeting SATB2. J Cell Biol 197: 509-521, 2012.

3. Dirckx N, Van Hul M and Maes C: Osteoblast recruitment to sites of bone formation in skeletal development, homeostasis, and regeneration. Birth Defects Res C Embryo Today 99: 170-191, 2013

4. Blagojevic M, Jinks C, Jeffery A and Jordan KP: Risk factors for onset of osteoarthritis of the knee in older adults: A systematic review and meta-analysis. Osteoarthritis Cartilage 18: 24-33, 2010.

5. Li J, Zhang Y, Zhao Q, Wang J and He X: MicroRNA-10a influences osteoblast differentiation and angiogenesis by regulating $\beta$-catenin expression. Cell Physiol Biochem 37: 2194-2208, 2015 .

6. Mattick JS: RNA regulation: A new genetics? Nat Rev Genet 5: 316-323, 2004

7. Chen L, Holmstrøm K, Qiu W, Ditzel N, Shi K, Hokland L and Kassem M: MicroRNA-34a inhibits osteoblast differentiation and in vivo bone formation of human stromal stem cells. Stem Cells 32: 902-912, 2014.

8. Chen H, Ji X, She F, Gao Y and Tang P: miR-628-3p regulates osteoblast differentiation by targeting RUNX2: Possible role in atrophic non-union. Int J Mol Med 39: 279-286, 2017.

9. Li Z, Hassan MQ, Volinia S, van Wijnen AJ, Stein JL, Croce CM, Lian JB and Stein GS: A microRNA signature for a BMP2-induced osteoblast lineage commitment program. Proc Natl Acad Sci USA 105: 13906-13911, 2008.

10. Liu X, Deng Y, Xu Y, Jin W and Li H: MicroRNA-223 protects neonatal rat cardiomyocytes and $\mathrm{H} 9 \mathrm{c} 2$ cells from hypoxia-induced apoptosis and excessive autophagy via the Akt/mTOR pathway by targeting PARP-1. J Mol Cell Cardiol 118: 133-146, 2018.

11. Qin D, Wang X, Li Y, Yang L, Wang R, Peng J, Essandoh K, Mu X, Peng T, Han Q, et al: MicroRNA-223-5p and -3p cooperatively suppress necroptosis in ischemic/reperfused hearts. J Biol Chem 291: 20247-20259, 2016.

12. Zhou W,PalAS,Hsu AY,GurolT,ZhuX,Wirbisky-HershbergerSE, Freeman JL, Kasinski AL and Deng Q: MicroRNA-223 suppresses the canonical NF- $\mathrm{B}$ pathway in basal keratinocytes to dampen neutrophilic inflammation. Cell Rep 22: 1810-1823, 2018.

13. Sugawara S, Yamada Y, Arai T, Okato A, Idichi T, Kato M, Koshizuka K, Ichikawa T and Seki N: Dual strands of the miR-223 duplex (miR-223-5p and miR-223-3p) inhibit cancer cell aggressiveness: Targeted genes are involved in bladder cancer pathogenesis. J Hum Genet 63: 657-668, 2018.
14. Berenstein R, Nogai A, Waechter M, Blau O, Kuehnel A, Schmidt-Hieber M, Kunitz A, Pezzutto A, Dörken B and Blau IW: Multiple myeloma cells modify VEGF/IL-6 levels and osteogenic potential of bone marrow stromal cells via Notch/miR-223. Mol Carcinog 55: 1927-1939, 2016.

15. Leuenberger C, Schuoler C, Bye H, Mignan C, Rechsteiner T, Hillinger S, Opitz I, Marsland B, Faiz A, Hiemstra PS, et al: MicroRNA-223 controls the expression of histone deacetylase 2: A novel axis in COPD. J Mol Med (Berl) 94: 725-734, 2016.

16. Lin X, Xiong D, Peng YQ, Sheng ZF, Wu XY, Wu XP, Wu F, Yuan LQ and Liao EY: Epidemiology and management of osteoporosis in the People's Republic of China: Current perspectives. Clin Interv Aging 10: 1017-1033, 2015.

17. Rau CS, Wu SC, Kuo PJ, Chen YC, Chien PC, Hsieh HY and Hsieh $\mathrm{CH}$ : Epidemiology of bone fracture in female trauma patients based on risks of osteoporosis assessed using the osteoporosis self-assessment tool for Asians score. Int J Environ Res Public Health 14: E1380, 2017

18. Lin J, Yang Y, Zhang X, Ma Z, Wu H, Li Y, Yang X, Fei Q and Guo A: BFH-OSTM, a new predictive screening tool for identifying osteoporosis in elderly Han Chinese males. Clin Interv Aging 12: 1167-1174, 2017.

19. Cherian KE, Kapoor N, Shetty S, Naik D, Thomas N and Paul TV: Evaluation of different screening tools for predicting femoral neck osteoporosis in rural South Indian postmenopausal women. J Clin Densitom 21: 119-124, 2018.

20. Zhu X, Luo J, Chen X, Wang J, Wang G, Li H, Xu Y, Feng J and $\mathrm{Tu} \mathrm{H}$ : Expression characteristic and significance of interleukin-6, nuclear factor kappa beta, and bone formation markers in rat models of osteoporosis. Transl Res 152: 18-23, 2008.

21. Hiligsmann M, Dellaert BG, Watson V and Boonen A: Comment on: Patients' preferences for anti-osteoporosis drug treatment: A cross-European discrete choice experiment: Reply. Rheumatology (Oxford) 57: 584-585, 2018.

22. Nogués X and Martinez-Laguna D: Update on osteoporosis treatment. Med Clin (Barc) 150: 479-486, 2018 (In English, Spanish).

23. Bushati N and Cohen SM: microRNA functions. Annu Rev Cell Dev Biol 23: 175-205, 2007.

24. Chua JH, Armugam A and Jeyaseelan K: MicroRNAs: Biogenesis, function and applications. Curr Opin Mol Ther 11: 189-199, 2009.

25. Foshay KM and Gallicano GI: Small RNAs, big potential: The role of MicroRNAs in stem cell function. Curr Stem Cell Res Ther 2: 264-271, 2007.

26. Ma Y, Yao N, Liu G, Dong L, Liu Y, Zhang M, Wang F, Wang B, Wei X, Dong H, et al: Functional screen reveals essential roles of miR-27a/24 in differentiation of embryonic stem cells. EMBO J 34: 361-378, 2015.

27. Zhang Y, Gao Y, Cai L, Li F, Lou Y, Xu N, Kang Y and Yang H: MicroRNA-221 is involved in the regulation of osteoporosis through regulates RUNX2 protein expression and osteoblast differentiation. Am J Transl Res 9: 126-135, 2017.

28. Chung AC, Huang XR, Meng X and Lan HY: miR-192 mediates TGF-beta/Smad3-driven renal fibrosis. J Am Soc Nephrol 21: 1317-1325, 2010.

29. Xie Y, Zhang L, Gao Y, Ge W and Tang P: The multiple roles of Microrna-223 in regulating bone metabolism. Molecules 20: 19433-19448, 2015.

30. Mader R, Sarzi-Puttini P, Atzeni F, Olivieri I, Pappone N, Verlaan JJ and Buskila D: Extraspinal manifestations of diffuse idiopathic skeletal hyperostosis. Rheumatology (Oxford) 48: 1478-1481, 2009

31. Verlaan JJ, Boswijk PF, de Ru JA, Dhert WJ and Oner FC: Diffuse idiopathic skeletal hyperostosis of the cervical spine: An underestimated cause of dysphagia and airway obstraction. Spine J 11: 1058-1067, 2011.

32. Kim SK, Lee MH and Rhee MH: Studies on the effects of biomedicinal agents on serum concentration of $\mathrm{Ca} 2+, \mathrm{P}$ and ALP activity in osteoporosis-induced rats. J Vet Sci 4: 151-154, 2003.

33. Yang X, Tao XA, Liang JQ, Huang YJ and Yang XP: The dynamic changes of circulating OCN+ cells versus insulinlike growth factor-I during primary healing of orthognathic surgeries. Oral Surg Oral Med Oral Pathol Oral Radiol 113: 734-740, 2012.

34. Wang H, Cui Y, Luan J, Zhou X, Li C, Li H, Shi L and Han J: MiR-5100 promotes osteogenic differentiation by targeting Tob2. J Bone Miner Metab 35: 608-615, 2017. 
35. Liu X, Xu H, Kou J, Wang Q, Zheng X and Yu T: MiR-9 promotes osteoblast differentiation of mesenchymal stem cells by inhibiting DKK1 gene expression. Mol Biol Rep 43: 939-946, 2016.

36. Swetha RG, Ramaiah S and Anbarasu A: Molecular dynamics studies on D835N mutation in FLT3-its impact on FLT3 protein structure. J Cell Biochem 117: 1439-1445, 2016.

37. Noh JH, Jung KH, Kim JK, Eun JW, Bae HJ, Xie HJ, Chang YG, Kim MG, Park WS, Lee JY and Nam SW: Aberrant regulation of HDAC2 mediates proliferation of hepatocellular carcinoma cells by deregulating expression of G1/S cell cycle proteins. PLoS One 6: e28103, 2011.
38. Jung KH, Noh JH, Kim JK, Eun JW, Bae HJ, Xie HJ, Chang YG, Kim MG, Park H, Lee JY and Nam SW: HDAC2 overexpression confers oncogenic potential to human lung cancer cells by deregulating expression of apoptosis and cell cycle proteins. J Cell Biochem 113: 2167-2177, 2012. 